\title{
Impaired White Matter Development in Extremely Low-Birth- Weight Infants with Previous Brain Hemorrhage
}

\author{
X. Ou, C.M. Glasier, R.H. Ramakrishnaiah, S.B. Mulkey, Z. Ding, T.L. Angtuaco, A. Andres, and J.R. Kaiser
} O- EBM

\begin{abstract}
BACKGROUND AND PURPOSE: Brain hemorrhage is common in premature infants. The purpose of the study is to evaluate white matter development in extremely low-birth-weight infants with or without previous brain hemorrhage.
\end{abstract}

MATERIALS AND METHODS: Thirty-three extremely low-birth-weight infants were prospectively enrolled and included in this institutional review board-approved study. Another 10 healthy term infants were included as controls. The medical records of the extremely low-birth-weight infants were reviewed for sonography diagnosis of intraventricular hemorrhage. All infants had an MR imaging examination at term-equivalent age for detection of previous hemorrhage, and their white matter was scored and compared among different groups. DTI measured fractional anisotropy values were also compared voxelwise by tract-based spatial statistics.

RESULTS: Compared with controls, the white matter score was not significantly different in extremely low-birth-weight infants without blood deposition on MR imaging ( $P=.17)$, but was significantly worse in extremely low-birth-weight infants with blood deposition on MR imaging but no intraventricular hemorrhage diagnosis by sonography $(P=.02)$, in extremely low-birth-weight infants with grade 1 or 2 intraventricular hemorrhage on sonography $(P=.003)$, and in extremely low-birth-weight infants with grade 3 or 4 intraventricular hemorrhage on sonography $(P=.0001)$. Extremely low-birth-weight infants without blood deposition on MR imaging did not show any white matter regions with significantly lower fractional anisotropy values than controls. Extremely low-birth-weight infants with blood deposition on MR imaging, but no intraventricular hemorrhage diagnosis, did show white matter regions with significantly lower fractional anisotropy values, and extremely low-birth-weight infants with intraventricular hemorrhage diagnosis had widespread white matter regions with lower fractional anisotropy values.

CONCLUSIONS: Previous brain hemorrhage is associated with abnormal white matter in extremely low-birth-weight infants at termequivalent age, and sonography is not sensitive to minor hemorrhages that are sufficient to cause white matter injury.

ABBREVIATIONS: ELBW = extremely low-birth-weight; FA = fractional anisotropy; IVH = intraventricular hemorrhage; TBSS = tract-based spatial statistics

$\mathbf{P}$ reterm birth and associated low birth weight remains a prevalent condition despite advances in obstetric and neonatal care. Of particular concern are infants born with extremely low birth weight (ELBW, birth weight $\leq 1000 \mathrm{~g}$ ). In addition to the

\footnotetext{
Received January 7, 2014; accepted after revision March 10.

From the Department of Radiology (X.O., C.M.G., R.H.R., T.L.A.), University of Arkansas for Medical Sciences, Little Rock, Arkansas; Departments Pediatrics (S.B.M., A.A.) and Pediatric Radiology (X.O., C.M.G., R.H.R.), Arkansas Children's Hospital, Little Rock, Arkansas; Vanderbilt University Institute of Imaging Sciences (Z.D.), Nashville, Tennessee; Arkansas Children's Nutrition Center (A.A.), Little Rock, Arkansas; and Departments of Pediatrics and Obstetrics and Gynecology, Section of Neonatology (J.R.K.), Baylor College of Medicine, Texas Children's Hospital, Houston, Texas.

Please address correspondence to Xiawei Ou, PhD, Slot 105, 1 Children's Way, Little Rock, AR 72202; e-mail: ouxiawei@uams.edu

O-- Indicates open access to non-subscribers at www.ajnr.org

Evidence-Based Medicine Level 2.

http://dx.doi.org/10.3174/ajnr.A3988
}

necessity for prolonged neonatal intensive care, poor neurodevelopmental outcome is quite common in surviving ELBW infants. ${ }^{1,2}$ Neurologic and developmental deficits have been associated with neonatal brain injury in preterm infants, especially injury to the cerebral white matter. ${ }^{3,4}$ White matter abnormalities are common in very preterm infants $s^{5,6}$ and can range from macroscopic injury such as cystic periventricular leukomalacia, which can be detected by sonography, to microstructural abnormalities that can only be measured by sensitive MR imaging methods such as DTI, ${ }^{7-9}$ and can persist from the neonatal age to adolescence, and then to adulthood. ${ }^{10,11}$

Intraventricular hemorrhage (IVH) is the most common form of brain hemorrhage in very preterm infants, and is frequently accompanied by white matter injury ${ }^{12}$ such as periventricular hemorrhagic infarction. ${ }^{13}$ IVH is conventionally graded 1 to 4 , ranging from subependymal germinal hemorrhage not extending 
Table 1: MRI findings for the 9 ELBW infants with normal ultrasounds with blood product deposition noted on MRI at term-equivalent age

\begin{tabular}{|c|c|c|}
\hline Subject & Location & Findings \\
\hline 1 & Occipital horn of the left lateral ventricle & Minimal non-acute blood products \\
\hline 2 & Bilateral choroid plexus & Minimal non-acute blood products \\
\hline 3 & Bilateral cerebellar hemispheres & Old hemorrhage \\
\hline 4 & $\begin{array}{l}\text { Subependymal regions of the lateral } \\
\text { ventricles }\end{array}$ & Old hemorrhage \\
\hline 5 & Subependymal regions and cerebellum & Old hemorrhage \\
\hline 6 & Bilateral frontal white matter and cerebellum & Old hemorrhage \\
\hline 7 & $\begin{array}{l}\text { Ependyma of the posterior right lateral } \\
\text { ventricle }\end{array}$ & Minimal non-acute blood products \\
\hline 8 & $\begin{array}{l}\text { Subependymal of the posterior right lateral } \\
\text { ventricle }\end{array}$ & Minimal non-acute blood products \\
\hline 9 & Left subependymal region & Minimal non-acute blood products \\
\hline
\end{tabular}

the Arkansas Children's Hospital neonatal intensive care units were recruited for MR imaging examinations. Parents of ELBW infants who were stable at near term-equivalent age were approached, and informed consent was obtained from those who agreed to participate in the MR imaging study. All procedures complied with local institutional review board regulations. ELBW infants with complex congenital anomalies, central nervous system malformations, chromosomal abnormalities, or hydrops fetalis were excluded. The medical records of the ELBW infants were reviewed for cranial sonogra- into ventricles to extended intraparenchymal hemorrhage with periventricular hemorrhagic infarction. While the adverse neurodevelopmental consequences of severe IVH (grade 3 or 4) are well recognized, the consequences of low-grade (grade 1 or 2) IVH need further study. For example, 1 study reported a higher percentage of neurodevelopmental impairment compared with controls in very preterm infants with grade 1 or $2 \mathrm{IVH},{ }^{14}$ whereas another study observed adverse neurodevelopmental outcome ${ }^{15}$ only when IVH was accompanied by white matter lesions. Because cystic periventricular leukomalacia rarely accompanies low-grade IVH, microstructural white matter injury will need further study. In addition, other types of brain hemorrhage, such as intracerebellar hemorrhages, though less frequent, may occur with or without IVH in preterm infants, and may also affect developing white matter.

While sonography is the standard method for the diagnosis of IVH and can detect overt white matter lesions, MR imaging provides better spatial resolution and higher contrast. Specific MR imaging pulse sequences such as susceptibility-weighted imaging and DTI are very sensitive to hemorrhage and white matter integrity, respectively, and can reveal pathology not apparent on sonography. While the ROI method is a conventional way to compare DTI parameters, such as the fractional anisotropy (FA) among subject groups, the recently developed tract-based spatial statistics (TBSS) method can provide a voxelwise statistical analysis of DTI parameters in the whole brain and is ROI-independent, thus nonsubjective. DTI-TBSS has shown great sensitivity in detecting white matter abnormalities in ELBW infants at termequivalent age. ${ }^{7,16}$

We hypothesized that any form of previous brain hemorrhage, including intracerebellar hemorrhage and all grades of IVH, may be associated with white matter microstructural abnormalities at term-equivalent age. To test our hypothesis, we performed MR imaging examinations at term-equivalent age in ELBW infants, reviewed sonography diagnosis of IVH, divided them into different groups according to the extent of hemorrhage, and compared their white matter development respectively to healthy term infants.

\section{MATERIALS AND METHODS Subjects}

ELBW infants with birth weight 401-1000 g (gestational age $<30$ weeks) from the University of Arkansas for Medical Sciences and phy diagnosis of IVH. Most of the ELBW infants had 2 cranial ultrasounds during the first week of life; if an abnormality was noted, serial cranial ultrasounds were performed during the initial hospitalization. Other ELBW infants had additional sonography screenings after the first week of life for other clinical reasons. In total, 39 ELBW infants were enrolled and completed an MR imaging examination. Among them, 1 infant did not have a valid DTI scan due to motion artifact and was therefore excluded from the analysis; 5 infants had moderate-to-severe ventricular dilation ( 4 of them had an sonography diagnosis of grade 3 or $4 \mathrm{IVH}$ ) on MR imaging examination and were also excluded because of the concern of large white matter structural distortion and the difficulty for image registration in the DTI-TBSS analysis. For the remaining 33 ELBW infants, 7 had grade 3 or 4 IVH (6 were diagnosed during the first week and 1 was diagnosed during the first month), 8 had grade 1 or 2 IVH (7 diagnosed during the first week and 1 diagnosed during the first month), 9 had no sonography diagnosis of IVH but showed blood product deposition on MR imaging at term-equivalent age (Table 1), and another 9 had no sonography diagnosis of IVH and also had no blood product deposition on MR imaging. In addition, 10 term healthy infants were recruited from another longitudinal clinical trial at our institution that follows healthy pregnant woman and their neonates. The parents consented soon after delivery for MR imaging examination of their neonates, and the MR imaging data served as control data to the MR imaging of ELBW infants. The postmenstrual ages at MR imaging were similar between the ELBW and the control infants. The subject characteristics, as well as the gestational age (based on menstrual dating) and postmenstrual age at MR imaging for the ELBW and control infants are listed in Table 2.

\section{MR Imaging Examinations}

All ELBW infants had MR imaging examinations without sedation at term-equivalent age. The infants were fed in the MR imaging suite 30 minutes before the scan, swaddled in warm sheets, and immobilized using a MedVac Infant Immobilizer (CFI Medical Solutions, Fenton, Michigan). The MR imaging examinations were performed on a 1.5T Achieva MR imaging scanner (Philips Health Care, Best, the Netherlands) with $60 \mathrm{~cm}$ bore size, 33 $\mathrm{mT} / \mathrm{m}$ gradient amplitude, and $100 \mathrm{mT} / \mathrm{m} / \mathrm{ms}$ maximum slew rate. A pediatric 8 -channel sensitivity encoding head coil was 
Table 2: Gestational and postmenstrual ages for control and ELBW infants

\begin{tabular}{|c|c|c|c|c|}
\hline & $\begin{array}{l}\text { Number of } \\
\text { Subjects }\end{array}$ & $\begin{array}{l}\text { Gestational Age } \\
\text { (weeks) }\end{array}$ & $\begin{array}{l}\text { Postmenstrual Age } \\
\text { at MRI (weeks) }\end{array}$ & $\begin{array}{l}P \text { Value of Postmenstrual } \\
\text { Age Compared with Controls }\end{array}$ \\
\hline Controls (term infants) & $n=10$ & $39.0 \pm 0.9$ & $41.2 \pm 1.0$ & $\mathrm{~N} / \mathrm{A}$ \\
\hline $\begin{array}{l}\text { ELBW infants without blood product deposition } \\
\text { on MRI }\end{array}$ & $n=9$ & $25.2 \pm 1.2$ & $40.1 \pm 1.0$ & .05 \\
\hline $\begin{array}{l}\text { ELBW infants with blood product deposition on } \\
\text { MRI but no IVH diagnosis }\end{array}$ & $n=9$ & $25.2 \pm 1.4$ & $40.4 \pm 1.1$ & .18 \\
\hline ELBW infants with grades 1 or 2 IVH diagnosis & $n=8$ & $24.7 \pm 1.2$ & $41.1 \pm 3.7$ & .51 \\
\hline ELBW infants with grades 3 or 4 IVH diagnosis & $n=7$ & $25.3 \pm 1.3$ & $40.4 \pm 2.7$ & .16 \\
\hline
\end{tabular}

a $P>.05$ for the age comparison between any ELBW groups.

used. A neonatal brain MR imaging protocol was used, which includes sagittal T1-weighted 3D (acquisition matrix $1 \mathrm{~mm} \times 1$ $\mathrm{mm} \times 1.5 \mathrm{~mm}$ ) reconstructed to 3 planes, axial T2-weighted, axial diffusion-weighted, and axial $\mathrm{T} 2 *$-weighted gradient-echo or SWI sequences (switched from gradient-echo to SWI at the early stage of this study for better sensitivity to blood product deposition). In addition, a single-shot spin-echo-planar imaging sequence with acquisition voxel size $2 \mathrm{~mm} \times 2 \mathrm{~mm} \times 3 \mathrm{~mm}$ and diffusion weighting gradients $\left(b=700\right.$ seconds $\left./ \mathrm{mm}^{2}\right)$ uniformly distributed in 15 directions was used to acquire DTI data. At approximately 2 weeks of age, the controls had a no-sedation MR imaging with the same experimental procedures and same T2weighted, DTI, and T1-weighted 3D sequences.

\section{White Matter Abnormality Scoring}

The conventional MR images (including T1-3D, axial T2, DWI, and gradient-echo or SWI) for all control and ELBW infants were transmitted to a PACS and were evaluated by 2 neuroradiologists (C.M.G., > 28 years of experience; and R.H.R., $>5$ years of experience in neuroradiology practice) who were blinded to the grouping information of the ELBW infants. They independently determined whether there was blood product deposition on the $\mathrm{T} 2{ }^{\star}$ gradient-echo or susceptibility-weighted images. Either "positive" or "negative" was recorded in the data sheet, with no grading of mild/moderate/severe. The radiologists also independently scored the white matter abnormality for each infant, using a method similar to that of Woodward et al. ${ }^{4}$ The scoring method consisted of 6 components: white matter signal intensity on T1 and T2, volume of periventricular white matter, presence of cysts, ventricular dilation, abnormality on DWI, and corpus callosum thickness, with each component scored from 1 to 4, corresponding to normal, mildly, moderately, and severely affected, respectively. The overall white matter abnormality score was the sum of the 6 sub-category scores, and the scores from the 2 neuroradiologists were averaged. A normal brain would have a score of 6 . The higher the score, the more severe were the white matter abnormalities. The average white matter abnormality score for each ELBW group and for the controls were calculated and compared. In addition, to investigate whether previous brain hemorrhage was a factor in white matter abnormality score at term-equivalent age in the ELBW infants, a 1-way ANOVA was used.

\section{DTI-TBSS Analysis}

The FA maps for each subject were computed from the scannercarried software (Fibertrak, Philips) and were exported to a workstation with the FMRIB Software Library for TBSS analysis (V1.2, http://fsl.fmrib.ox.ac.uk/fsl/fslwiki/TBSS/UserGuide) by an MR imaging physicist with $>5$ years of experience in DTI data analysis. The FA images were first preprocessed. Each FA dataset was aligned to every other one to identify the most representative set that had the least amount of total warping; this one subsequently served as the target FA. Nonlinear transforms were then performed to register each FA dataset to the target. Standard nonlinear transformation program in TBSS V1.2 was used, except for modification to reflect registration of all FA to the target FA from our own data but not to the Montreal Neurological Institute 152 template. Afterward, all FA images were merged, averaged, and entered into the FA skeletonization program to create a mean FA skeleton in which a threshold of FA $\geq 0.15$ was selected. Ventriculomegaly did not affect the TBSS registration and skeletonization because infants with moderate to severe ventricular dilation (as defined by conventional MR imaging white matter grading) were already excluded. Nevertheless, the registration and the skeletonization were carefully reviewed section by section to ensure no misregistration. Finally, randomization with threshold-free cluster enhancement was used to perform voxelwise comparison of FA values in each ELBW group versus the control group collectively.

\section{Statistics}

For the comparison of ages and white matter abnormality scores between groups, values were reported as mean \pm standard deviation and a Wilcoxon rank sum test was performed by Matlab software (MathWorks, Natick, Massachusetts) to determine if there was difference. $P<.05$ was regarded as significant. In addition, Cohen $\kappa$ coefficients were used to measure the interrater agreement of white matter scoring and hemorrhage determination by the 2 neuroradiologists. The $\kappa$ coefficients were interpreted as follows ${ }^{17}: 0.41-0.60$, moderate agreement; $0.61-0.80$, substantial agreement; $0.81-0.99$, almost perfect agreement; and 1 , perfect agreement. For the effects of previous hemorrhage on white matter abnormality scores in ELBW infants, a 1-way ANOVA was performed by Matlab. For the DTI-TBSS analysis, $P<.05$ after multiple comparisons correction controlling for family wise error was used for the voxelwise comparison in FSL to detect regions with significant difference in FA values.

\section{RESULTS}

There was no difference in birth gestational age between the ELBW infant groups and no difference in postmenstrual age at MR imaging between the ELBW infant groups. The postmenstrual age at MR imaging was similar in controls and the ELBW infants groups (Table 2).

AJNR Am J Neuroradiol 35:1983-89 Oct 2014 www.ajnr.org 


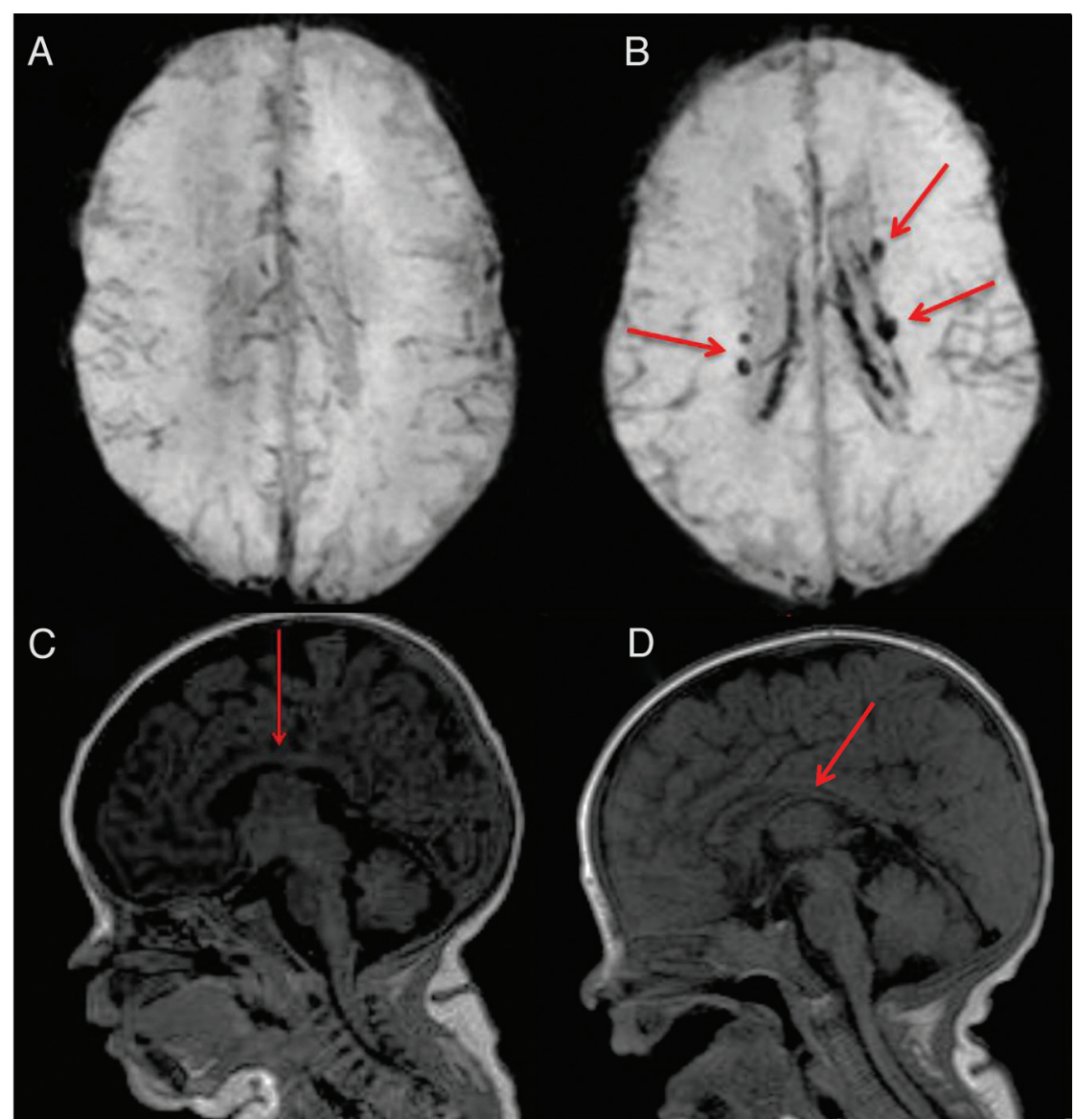

FIG 1. Examples of conventional MR imaging findings: $A, S W I$ shows brain without blood deposition in an ELBW infant; $B$, SWI shows brain with blood deposition (arrows show the old blood product) in an ELBW infant with sonography IVH diagnosis; $C$, normal thickness of corpus callosum (arrow) in an ELBW infant; $D$, moderate thinning of corpus callosum (arrow) in an ELBW infant.

Table 3: White matter abnormality scores for the control and ELBW infants

\begin{tabular}{lcc}
\hline & $\begin{array}{c}\text { White Matter } \\
\text { Abnormality } \\
\text { Score }\end{array}$ & $\begin{array}{c}\text { P Value } \\
\text { (Compared } \\
\text { with Controls) }\end{array}$ \\
\hline $\begin{array}{l}\text { Controls (term infants) } \\
\text { ELBW infants without blood } \\
\text { product deposition on MRI }\end{array}$ & $6.0 \pm .0$ & N/A \\
$\begin{array}{l}\text { ELBW infants with blood product } \\
\text { deposition on MRI but no IVH } \\
\text { diagnosis }\end{array}$ & $6.7 \pm .9$ & .17 \\
$\begin{array}{l}\text { ELBW infants with grades 1 or 2 } \\
\text { IVH diagnosis }\end{array}$ & $7.5 \pm 1.7$ & .02 \\
$\begin{array}{l}\text { ELBW infants with grades 3 or } 4 \\
\text { IVH diagnosis }\end{array}$ & $8.6 \pm 1.3$ & .003 \\
\hline
\end{tabular}

The interrater agreement by the 2 neuroradiologists on blood product deposition identified on MR imaging was perfect $(\kappa=1)$. The interrater agreement was substantial on white matter abnormality scores $(\kappa=0.71)$. Specifically, perfect agreement on presence of cysts $(\kappa=1)$ and on abnormality on DWI $(\kappa=1)$, almost perfect agreement on volume of periventricular white matter $(\kappa=0.83)$, substantial agreement on ventricular dilation $(\kappa=$ $0.80)$ and on white matter signal intensity on T1 and T2 $(\kappa=$ $0.69)$, and moderate agreement on corpus callosum thickness $(\kappa=0.52)$. Figure 1 shows examples of blood product deposition and white matter grading (for the component of corpus callosum thickness). White matter abnormality scores for the control and ELBW infants are reported in Table 3. The white matter abnormality score was $6.0 \pm 0.0$ for the controls, $6.2 \pm$ 0.3 for the ELBW infants without old blood deposition on MR imaging at termequivalent age, $6.7 \pm 0.9$ for ELBW infants with old blood on MR imaging but no IVH diagnosis by sonography, $7.5 \pm$ 1.7 for ELBW infants with grade 1 or 2 $\mathrm{IVH}$, and $8.6 \pm 1.3$ for ELBW infants with grade 3 or 4 IVH. Compared with controls, the white matter abnormality score for ELBW infants without old blood on MR imaging was not significantly different $(P=.17)$, but was significantly different in the other ELBW infant groups ( $P=$ $.02, .003$, and .0001 , respectively). A boxplot of the white matter abnormality score for the 4 ELBW infant groups is shown in Fig 2. One-way ANOVA of these data revealed a significant effect $(P<$ $.001)$ of previous hemorrhage on white matter abnormality score by MR imaging at term-equivalent age.

Figure 3 shows the DTI-TBSS results for the ELBW and control infants. Compared with controls, ELBW infants without old blood deposition on MR imaging at term-equivalent age did not show any white matter region with significantly lower FA values $(P<.05$, corrected). ELBW infants with old blood on MR imaging but no IVH diagnosis after birth showed a few regions with significantly lower FA values $(P<.05$, corrected). These regions were limited mostly to the splenium of the corpus callosum and the optic radiation. ELBW infants with grade 1 or 2 IVH diagnosis by sonography had widespread regions with lower FA values $(P<.05$, corrected), including the optic radiation, the genu, body, and splenium of the corpus callosum, the cingulum, and the frontal corona radiata. ELBW infants with grade 3 or $4 \mathrm{IVH}$ had similar but even more extensive white matter regions with decreased FA $(P<.05$, corrected), involving association, projection, and callosal fibers.

\section{DISCUSSION}

Our results demonstrate that previous hemorrhage in the brain had a significant effect on white matter development in ELBW infants at term-equivalent age. Compared with controls, ELBW infants with no apparent history of brain hemorrhage (as reflected by no blood product deposition on MR imaging) did not have a significant difference in white matter abnormality score or DTImeasured FA values in any brain regions; on the other hand, ELBW infants with signs of previous brain hemorrhage had significantly worse white matter scores and significantly lower FA values in many brain regions. Many of the ELBW infants without 
cranial sonography IVH diagnosis but with positive MR imaging findings of previous hemorrhage at term-equivalent age showed only minimal blood product deposition near the lateral ventricles on MR imaging. This could represent a minor intracranial hem-

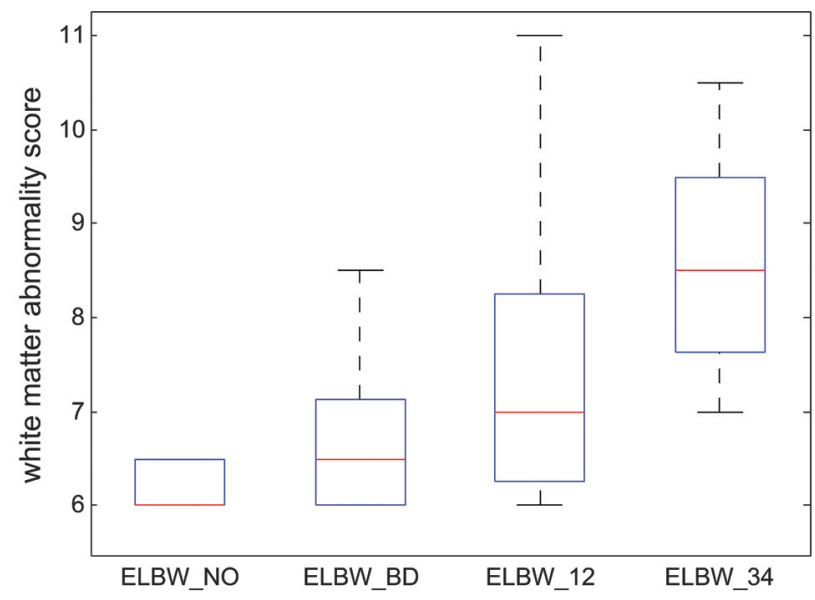

FIG 2. Boxplot of the white matter abnormality scores for the 4 ELBW infant groups show that the more previous brain hemorrhage, the more white matter abnormality at term-equivalent age. A score of 6 means normal white matter. Any score greater than 6 means abnormality. The line in the middle for each box is the median score for each group, and the box contains scores in 25-75 percentiles. Minimum/maximum scores in each group are also marked. A 1-way ANOVA revealed the significant effect $(P<.001)$ of brain hemorrhage on white matter abnormality score at term-equivalent age in ELBW infants. ELBW NO: ELBW infants without old blood product deposition on MR imaging at term-equivalent age; ELBW_BD: ELBW infants with old blood product on MR imaging but no IVH diagnosis by sonography; ELBW_12: ELBW infants with grades 1 or 2 IVH diagnosis; ELBW_34: ELBW infants with grades 3 or 4 IVH diagnosis.
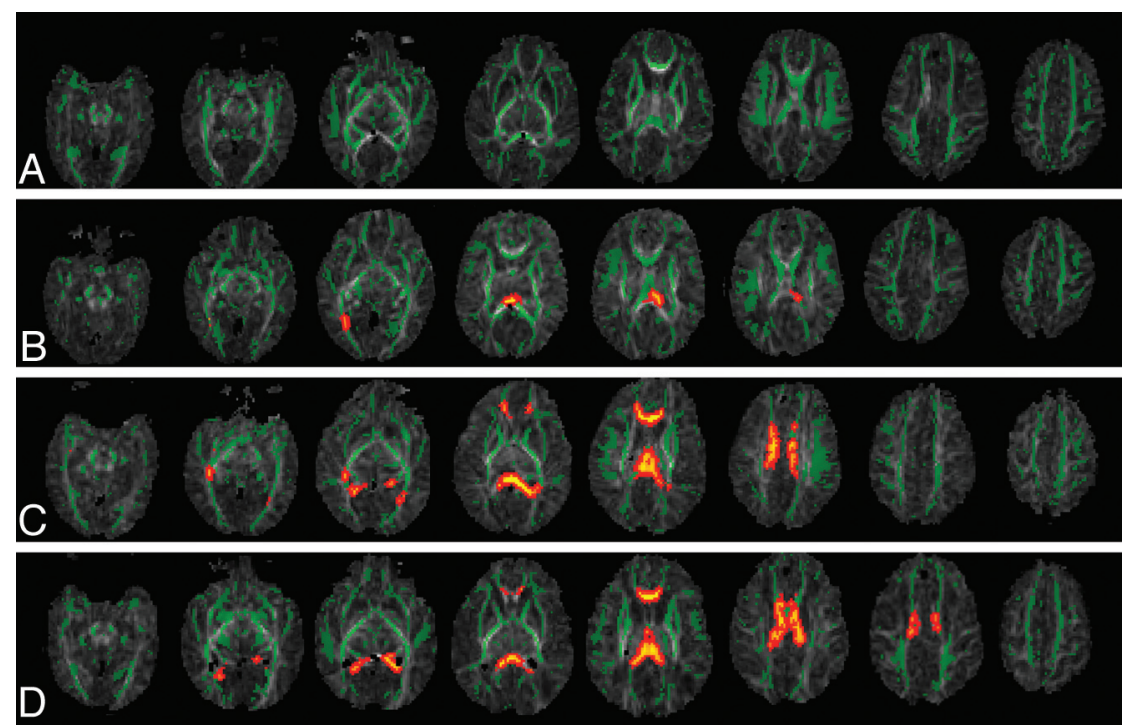

FIG 3. DTI-TBSS results show that the more previous brain hemorrhage, the more white matter microstructural abnormalities (as reflected by more regions with lower FA compared with controls). Green represents the average white matter skeleton overlaid on FA images, orange/ yellow represent white matter regions with significantly lower FA values (when compared with control infants, $P<.05$, fully corrected for multiple comparisons) in A, ELBW infants with no old blood product deposition on MR imaging at term-equivalent age, $B$, ELBW infants with old blood product deposition on MR imaging but no sonography IVH diagnosis after birth, C, ELBW infants with old blood product deposition on MR imaging and previous sonography diagnosis of grades 1 or $2 \mathrm{IVH}$, and D, ELBW infants with old blood product deposition on MR imaging and previous sonography diagnosis of grades 3 or $4 \mathrm{IVH}$. orrhage, which was not detected by early sonography screenings. Assuming that infants with blood on MR imaging and not on white matter score and more regions with lower FA values.

the association of IVH with poor neurodevelopmenta on severe IVH study showed that grade 3-4 IVH was significantly associated with neurodevelopmental morbidity in ELBW infants at 18-22 months corrected age, whereas grade $1-2$ IVH was not considered. ${ }^{18}$ In another large study of infants born at $<28$ weeks gesta, the risk of developing cerebral palsy at 2 years of age was only responding to grade 1 or 2) compared with those with normal neonatal sonography findings; however, this risk was much (39\%) in those with ventriculomegaly. ${ }^{19}$ In contrast, anscores and higher rates of neurodevelopmental impairment or major neurologic abnormality at 20 months corrected age compared with ELBW infants with normal cranial ultrasounds. ${ }^{20}$ Furthermore, another study showed that $9 \%$ of ELBW infants with normal neonatal ultrasounds developed cerebral palsy and 25\% had Mental Developmental Index scores $<70$ at 18-22 months age. $^{21}$ These studies suggest that all grades of IVH may be a risk factor of poor neurodevelopmental outcome in ELBW infants, and normal cranial sonography findings do not necessarily correlate with normal neurodevelopment.

The percentage of missed brain hemorrhage diagnosis by cranial sonography was high in our study $(27 \%)$. This was likely because of these being minimal hemorrhages that would be undetectable by sonography, and less likely (though still possible) due to hemorrhages that occurred during the interval between the last sonography screening and the MR imaging examination at term-equivalent age as most hemorrhages occur during the first few days of life for premature infants and our sonography screening covered at least the first week of life. Cerebellar hemorrhage by MR imaging was also observed in 3 ELBW infants without hemorrhage noted on sonography, which is a less recognized area of brain injury ${ }^{22}$ but has recently been shown to be associated with long-term neurodevelopmental disabilities in premature infants. ${ }^{22,23}$ Overall, as our study indicated, MR imaging (particularly the $\mathrm{T} 2^{\star}$-weighted sequences) was more sensitive to brain hemorrhage and may reveal pathologic 
findings that cannot be diagnosed by cranial sonography. There is currently no large-scale study (compared with sonography studies with large sample sizes) to evaluate the association of MR imaging findings of hemorrhage at term-equivalent age and neurodevelopmental outcome at later age; MR imaging is not routinely performed and not a standard of care in ELBW infants in most centers. Our results showed that the ELBW infants with normal ultrasounds but with blood product deposition on MR imaging also had significant white matter abnormalities at term-equivalent age. Because white matter development is known to be most predictive of neurodevelopmental outcome, ${ }^{3,4}$ our MR imaging findings of additional ELBW infants with hemorrhagic injury and white matter abnormalities indicate that MR imaging screening at term-equivalent age in ELBW infants may be beneficial to identify those infants with a higher risk of poor neurodevelopmental outcome.

The exact mechanism of the observed association between earlier brain hemorrhage and later white matter abnormality at termequivalent needs to be determined. It is believed that some white matter injuries are the direct result of IVH. ${ }^{13}$ The germinal matrix destruction caused by IVH may induce deficits of white matter microstructural development because the germinal matrix provides precursor cells that become oligodendroglia and astrocytes, which are important for myelination. More severe IVH may also cause periventricular venous congestion, which generates ischemia and results in periventricular hemorrhagic infarction in white matter. ${ }^{13}$ Other factors such as free radical formation induced by iron from red blood cells and cellular effects by cytokines may also link hemorrhage to white matter injury. ${ }^{24}$

One limitation of our study is that we excluded ELBW infants with moderate to severe ventricular dilation at term-equivalent age in the DTI-TBSS analysis to avoid imaging registration artifacts. Most of these ELBW infants had grades 3 or 4 IVH diagnosed by sonography within the first week of life. Our sample for the grade 3 or 4 IVH group therefore did not include all available subjects. Because severe ventricular dilation worsens the white matter score (if we include these ELBW infants, the white matter abnormality scores for the grade 3 or 4 IVH group were higher: $10.2 \pm 3.1, P<.0001$ compared with controls) and DTI has shown white matter microstructural abnormalities in infants with ventriculomegaly, ${ }^{25}$ we do not feel the exclusion of these infants altered the conclusions of our study. Meanwhile, our DTI-TBSS analysis provided a nonsubjective region-specific quantitative measure of white matter; this is a great addition to (and maybe more sensitive than) the semiquantitative but more clinically feasible white matter scoring. For better sensitivity to small hemorrhages in ELBW infants, we switched from gradient-echo $(n=12$, $67 \%$ with positive findings) to SWI sequences ( $n=21,76 \%$ with positive findings). Reviewing several MR imaging datasets with both gradient-echo and SWI sequences acquired during the transition showed that gradient-echo may be adequate for the determination of whether there was blood product deposition; however, it is still possible that there were small hemorrhages omitted by the gradient-echo images. Another limitation is the relatively small sample size in this study. Nevertheless, our results achieved statistical significance $(P<.05$ for TBSS, after multiple comparison correction). Finally, lack of data correlating MR imagingrevealed hemorrhage and white mater injury to neurodevelopmental outcome is another limitation that cannot be addressed in this study. Further study is needed to determine whether hemorrhage noted by MR imaging at term-equivalent age is a valid predictor of long-term neurodevelopmental outcome.

\section{CONCLUSIONS}

In this study, we showed that previous hemorrhage in the brain had significant effects on white matter development in ELBW infants at term-equivalent age. Compared with controls, ELBW infants with no blood product deposition in the brain did not have a significant difference in white matter abnormality score or microstructural development measured by DTI, whereas ELBW infants with signs of previous brain hemorrhage had significantly worse white matter scores and lower FA values in many regions in the brain. Notably, cranial sonography after birth was not sensitive to amounts of hemorrhage that were sufficient to cause white matter injury at term-equivalent age. Our results suggest that MR imaging at term-equivalent age may add value in determining neurodevelopmental outcome in ELBW infants who have normal ultrasounds.

\section{ACKNOWLEDGMENTS}

The assistance from ACH MR imaging technologists is gratefully appreciated. The help from research coordinator Patricia M. Brady, the advice from Dr. Thomas M. Badger, and the statistical consulting from Dr. Mario A. Cleves are also greatly appreciated.

Disclosures: Xiawei Ou was supported by the Children's University Medical Group at UAMS, the Thrasher Research Fund, and the International Society for Magnetic Resonance in Medicine. Jeffrey R. Kaiser was supported by the National Institutes of Health (1K23NS43185, RR20146, and 1R01NS060674), the NINDS, and the UAMS Translational Research Institute (1ULIRR029884). Sarah B. Mulkey was supported by the Center for Translational Neuroscience award from the National Institutes of Health (P20 GM103425). Aline Andres was supported by a grant from the USDA paid to her institution, unrelated to the current study.

\section{REFERENCES}

1. Marlow N, Wolke D, Bracewell MA, et al. Neurologic and developmental disability at six years of age after extremely preterm birth. N Engl J Med 2005;352:9-19

2. Wood NS, Marlow N, Costeloe K, et al. Neurologic and developmental disability after extremely preterm birth. $N$ Engl $\mathrm{J}$ Med 2000;343:378-84

3. Perlman JM. White matter injury in the preterm infant: an important determination of abnormal neurodevelopment outcome. Early Hum Dev 1998;53:99-120

4. Woodward LJ, Anderson PJ, Austin NC, et al. Neonatal MRI to predict neurodevelopmental outcomes in preterm infants. $N$ Engl J Med 2006;355:685-94

5. Larroque B, Marret S, Ancel PY, et al. White matter damage and intraventricular hemorrhage in very preterm infants: the EPIPAGE study. J Pediatr 2003;143:477-83

6. Volpe JJ. Cerebral white matter injury of the premature infantmore common than you think. Pediatrics 2003;112:176-80

7. Anjari M, Srinivasan L, Allsop JM, et al. Diffusion tensor imaging with tract-based spatial statistics reveals local white matter abnormalities in preterm infants. Neuroimage 2007;35:1021-27

8. Volpe JJ. Brain injury in premature infants: a complex amalgam of destructive and developmental disturbances. Lancet Neurol 2009; 8:110-24

9. Perlman JM, Risser R, Broyles RS. Bilateral cystic periventricular leukomalacia in the premature infant: associated risk factors. Pediatrics 1996;97:822-27

10. Eikenes L, Lohaugen GC, Brubakk AM, et al. Young adults born 
preterm with very low birth weight demonstrate widespread white matter alterations on brain DTI. Neuroimage 2011;54:1774-85

11. Skranes J, Vangberg TR, Kulseng S, et al. Clinical findings and white matter abnormalities seen on diffusion tensor imaging in adolescents with very low birth weight. Brain 2007;130:654-66

12. Kuban K, Sanocka U, Leviton A, et al. White matter disorders of prematurity: association with intraventricular hemorrhage and ventriculomegaly. J Pediatr 1999;134:539-46

13. Volpe JJ. Neurology of the Newborn, 4th ed. Philadelphia: W.B. Saunders; 2001

14. Klebermass-Schrehof K, Czaba C, Olischar M, et al. Impact of lowgrade intraventricular hemorrhage on long-term neurodevelopmental outcome in preterm infants. Childs Nerv Syst 2012;28: 2085-92

15. O'Shea TM, Allred EN, Kuban KCK, et al. Intraventricular hemorrhage and developmental outcomes at 24 months of age in extremely preterm infants. J Child Neurol 2012;27:22-29

16. Smith SM, Jenkinson M, Johansen-Berg H, et al. Tract-based spatial statistics: voxelwise analysis of multi-subject diffusion data. Neuroimage 2006;31:1487-505

17. Landis JR, Koch GG. Measurement of observer agreement for categorical data. Biometrics 1977;33:159-74

18. Vohr BR, Wright LL, Dusick AM, et al. Neurodevelopmental and functional outcomes of extremely low birth weight infants in the
National Institute of Child Health and Human Development Neonatal Research Network, 1993-1994. Pediatrics 2000;105: $1216-26$

19. Kuban KCK, Allred EN, O'Shea TM, et al. Cranial ultrasound lesions in the NICU predict cerebral palsy at age 2 years in children born at extremely low gestational age. J Child Neurol 2009;24:63-72

20. Patra K, Wilson-Costello D, Taylor HG, et al. Grades I-II intraventricular hemorrhage in extremely low birth weight infants: effects on neurodevelopment. J Pediatr 2006;149:169-73

21. Laptook AR, O'Shea TM, Shankaran S, et al. Adverse neurodevelopmental outcomes among extremely low birth weight infants with a normal head ultrasound: prevalence and antecedents. Pediatrics 2005; 115:673-80

22. Volpe JJ. Cerebellum of the premature infant: rapidly developing, vulnerable, clinically important. J Child Neurol 2009;24:1085-104

23. Limperopoulos C, Bassan H, Gauvreau K, et al. Does cerebellar injury in premature infants contribute to the high prevalence of longterm cognitive, learning, and behavioral disability in survivors? $P e-$ diatrics 2007;120:584-93

24. Bassan H. Intracranial hemorrhage in the preterm infant: understanding it, preventing it. Clin Perinatol 2009;36:737-62

25. Gilmore JH, Smith LC, Wolfe HM, et al. Prenatal mild ventriculomegaly predicts abnormal development of the neonatal brain. Biol Psychiatry 2008;64:1069-76 